This is an electronic reprint of the original article. This reprint may differ from the original in pagination and typographic detail.

Author(s): Tirronen, Maria; Banichuk, Nikolay; Jeronen, Juha; Saksa, Tytti; Tuovinen, Tero

Title: $\quad$ Stochastic analysis of the critical velocity of an axially moving cracked elastic plate

Year: $\quad 2014$

Version:

Please cite the original version:

Tirronen, M., Banichuk, N., Jeronen, J., Saksa, T., \& Tuovinen, T. (2014). Stochastic analysis of the critical velocity of an axially moving cracked elastic plate. Probabilistic engineering mechanics, 37(July), 16-23.

https://doi.org/10.1016/j.probengmech.2014.04.001

All material supplied via JYX is protected by copyright and other intellectual property rights, and duplication or sale of all or part of any of the repository collections is not permitted, except that material may be duplicated by you for your research use or educational purposes in electronic or print form. You must obtain permission for any other use. Electronic or print copies may not be offered, whether for sale or otherwise to anyone who is not an authorised user. 


\section{Author's Accepted Manuscript}

Stochastic analysis of the critical velocity of an axially moving cracked elastic plate

M. Tirronen, N. Banichuk, J. Jeronen, T. Saksa, T. Tuovinen

PROBABILISTIC EN G INEER IN G

M E C H A N I C S

Editor: P.D. Spanos

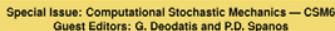

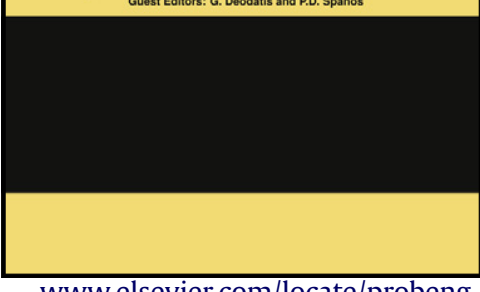

www.elsevier.com/locate/probeng-

PII:

S0266-8920(14)00026-5

DOI: $\quad$ http://dx.doi.org/10.1016/j.probengmech.2014.04.001

Reference: PREM2787

To appear in: Probabilistic Engineering Mechanics

Received date: 4 November 2013

Accepted date: 4 April 2014

Cite this article as: M. Tirronen, N. Banichuk, J. Jeronen, T. Saksa, T. Tuovinen, Stochastic analysis of the critical velocity of an axially moving cracked elastic plate, Probabilistic Engineering Mechanics, http://dx.doi.org/10.1016/j.probengmech.2014.04.001

This is a PDF file of an unedited manuscript that has been accepted for publication. As a service to our customers we are providing this early version of the manuscript. The manuscript will undergo copyediting, typesetting, and review of the resulting galley proof before it is published in its final citable form. Please note that during the production process errors may be discovered which could affect the content, and all legal disclaimers that apply to the journal pertain. 


\title{
Stochastic analysis of the critical velocity of an axially moving cracked elastic plate
}

\author{
M. Tirronen ${ }^{*, a}$, N.Banichuk ${ }^{\mathrm{a}, \mathrm{b}}$, J. Jeronen ${ }^{\mathrm{a}}$, T. Saksa ${ }^{\mathrm{a}}$, T.Tuovinen ${ }^{\mathrm{a}}$ \\ ${ }^{a}$ Department of Mathematical Information Technology, P.O. Box 35 (Agora), FI-40014 \\ University of Jyväskylä, Finland \\ ${ }^{b}$ Institute for Problems in Mechanics RAS, Prospect Vernadskogo 101, Bld. 1, 119526 \\ Moscow, Russian Federation
}

\section{Abstract}

In this study, a probabilistic analysis of the critical velocity for an axially moving cracked elastic and isotropic plate is presented. Axially moving materials are commonly used in modelling of manufacturing processes, like paper making and plastic forming. In such systems, the most serious threats to runnability are instability and material fracture, and finding the critical value of velocity is essential for efficiency. In this paper, a formula for the critical velocity is derived under constraints for the probabilities of instability and fracture. The significance of randomness in different model parameters is investigated for parameter ranges typical of paper material and paper machines. The results suggest that the most significant factors are variation in the crack length and tension magnitude.

Key words: Uncertainty, fracture, stability, moving material, plate, paper

\footnotetext{
${ }^{*}$ Corresponding author.

Email addresses: maria.j.e.tirronen@jyu.fi (M. Tirronen), juha.jeronen@jyu.fi (J. Jeronen), tytti.saksa@jyu.fi (T. Saksa), tero.tuovinen@jyu.fi (T.Tuovinen)
} 


\section{Introduction}

In industry, there are many systems the behaviour of which can be described by the mathematical model of an axially moving material. Thus, during the last few decades, the mechanics of such materials have aroused much interest among researchers. Traditionally, the studies of axially moving materials are based on a deterministic approach, although in reality, the problem parameters are not known deterministically. In industrial paper manufacturing, which is one of the application areas of axially moving materials, uncertainty factors include, e.g., the strength of the paper web, variation of tension with respect to space and time in the press system, and defects, which vary in their geometry and location in the web. These factors are considerable: according to Uesaka [1], the majority of web breaks in paper production are caused by tension variations, combined with strength variations of the paper web. Wathén [2] discusses the effect of flaws of paper on web breaks and, according to him, even a seemingly flawless paper can fail at very low tensions due to stress concentrations caused by discontinuities, e.g. cuts and shives, in structure.

Finding the optimal value of velocity for an axially moving material is essential, when the efficiency of the corresponding manufacturing process is considered. The most critical threats to good runnability of such a system are instability and material fracture, and on these phenomena a change in tension magnitude has opposite effects. An increase in tension has a stabilizing effect [3], but high tension may lead to growing or arising of cracks. Web tension too low or too high may cause a web break, which deteriorates production efficiency. 
The modelling of vibrations of travelling elastic materials has interested many researchers. The first paper on the subject dates from 1897, when Skutch published a paper [4] concerning the axially moving string. The first papers in English were published in the 1950's, when Sack [5] and Archibald and Emslie [6] studied the axially moving string model. Since then, many researchers have continued the studies of moving elastic material. E.g., Wickert and Mote [7] studied stability of axially moving strings and beams using modal analysis and Greens function method. The stability of travelling twodimensional rectangular membranes and plates has been studied, e.g., by Lin [8] and Banichuk et al. [3]. A more extensive literature review of the history of the studies concerning deterministic elastic models can be found in [3], the results of which we also exploit in this study. In the recent studies concerning axially moving plates, material properties such as orthotropicity $[9,10]$ or viscoelasticity $[11,12]$ have been taken into consideration and their effects on the plate behavior have been investigated.

In addition, there are studies considering stationary plates with random parameters. For example, the free transverse vibrations of elastic rectangular plates with random material properties were considered and statistical characteristics of the random eigenvalues were determined by Sobczyk [13]. Wood and Zaman [14] considered a collection of elastic rectangular plates with random inhomogeneities vibrating freely under simply supported boundary conditions. Soares [15] considered uncertainty modelling of plates subjected to compressive loads.

The field of fracture mechanics was developed by Irwin [16], based on the early papers of Inglis [17], Griffith [18] and Westergaard [19]. Various 
deterministic analysis of vibrations and stability of stationary cracked beams and plates exist in the literature. For a literature review, we refer to [20].

As far as the authors know, axially moving materials have been studied in a stochastic setup only in $[21,22]$. In these studies, the critical velocity of an axially moving plate was derived in the case in which there is a random length crack on the plate, or the tension, to which the plate is subjected, varies randomly. This research extends these studies by introducing several other parameters as random variables simultaneously in the model. In this paper, we also compare the effect of introducing variation between different problem parameters, in order to decide the randomness of which parameters is the most significant in terms of the critical velocity. For the analysis, we have chosen the setup and parameter ranges to be applicable for paper material and paper making.

The formula for the critical velocity of the plate is derived under constraints for instability and fracture. Depending on the distributions of the problem parameters, numerical methods may be needed in solving the critical velocity. In the paper industrial example, simultaneously introducing several problem parameters as random leads to the use of numerical methods. Due to its simplicity and accuracy, we use Monte Carlo simulation to solve the problem with several random variables.

\section{Critical velocity of a travelling plate}

We consider a rectangular part of an elastic and isotropic band, which is moving at a constant velocity $V_{0}$ between supporting rollers. Denoting the 
part as

$$
\mathcal{D}=\{(x, y): \quad 0<x<\ell, \quad-b<y<b\}
$$

where $\ell$ and $b$ are prescribed parameters of length and width, the plate is assumed to travel in the $x$ direction. The supporting rollers are located at $x=0$ and $x=\ell$. (See Figure 1.)

The considered part $\mathcal{D}$ is represented as a thin elastic plate having constant thickness $h$, Poisson ratio $\nu$, Young modulus $E$, and bending rigidity

$$
D=\frac{E h^{3}}{12\left(1-\nu^{2}\right)} \text {. }
$$

The mass of the plate per unit area is denoted by $m$. It is further assumed that the plate is subjected to homogeneous tension $T$ acting in the $x$ direction.

We consider the case in which there is a single crack in the plate. The length of the crack is denoted by $\xi$. (See also Figure 1.)

\subsection{Characterization of instability of the plate}

We first briefly present a deterministic stability analysis for a travelling plate without a crack. Especially, we are interested in critical regimes, where the plate approaches its maximum stable velocity. Details of the analysis can be found in [3].

We perform a standard dynamic analysis (see, e.g., [23]). The transverse displacement of the travelling plate is described by the deflection function $w$, which depends on the space coordinates $x, y$ and time $t$. It is assumed that the absolute values of the deflection function $w$ and its derivatives are small. The Kirchhoff plate theory is applied. To study the dynamic behavior of the plate, the following equation for the travelling plate is used:

$$
\frac{\partial^{2} w}{\partial t^{2}}+2 V_{0} \frac{\partial^{2} w}{\partial x \partial t}+\left(V_{0}^{2}-C^{2}\right) \frac{\partial^{2} w}{\partial x^{2}}+\frac{D}{m} \Delta^{2} w=0
$$


where

$$
\begin{aligned}
C & =\sqrt{\frac{T}{m}} \quad \text { and } \\
\Delta^{2} w & =\frac{\partial^{4} w}{\partial x^{4}}+\frac{\partial^{4} w}{\partial x^{2} \partial y^{2}}+\frac{\partial^{4} w}{\partial y^{4}} .
\end{aligned}
$$

As boundary conditions, the classical simply-supported and free boundary conditions $[24,25]$ are used. The simply supported boundary conditions read as

$$
(w)_{x=0, \ell}=0, \quad\left(\frac{\partial^{2} w}{\partial x^{2}}\right)_{x=0, \ell}=0, \quad-b \leq y \leq b
$$

and the equations for the boundaries free of tractions can be presented as follows:

$$
\begin{gathered}
\left(\frac{\partial^{2} w}{\partial y^{2}}+\nu \frac{\partial^{2} w}{\partial x^{2}}\right)_{y= \pm b}=0, \quad 0 \leq x \leq \ell, \\
\left(\frac{\partial^{3} w}{\partial y^{3}}+(2-\nu) \frac{\partial^{3} w}{\partial x^{2} \partial y}\right)_{y= \pm b}=0, \quad 0 \leq x \leq \ell .
\end{gathered}
$$

The solution of the dynamic boundary value problem of $(3)-(8)$ can be represented as

$$
w(x, y, t)=W(x, y) e^{i \tilde{\omega} t}=W(x, y) e^{\tilde{s} t}
$$

where $\tilde{\omega}$ is the frequency of small transverse vibrations and $\tilde{s}=i \tilde{\omega}$ is the complex characteristic parameter; $\tilde{s}=\operatorname{Re} \tilde{s}+i \operatorname{Im} \tilde{s}$.

If the parameter $\tilde{s}$ is purely imaginary and $\tilde{\omega}$ is real, the plate performs harmonic vibrations of a small amplitude and its motion can be considered stable. If the real part of $\tilde{s}$ becomes positive, the transverse vibrations grow exponentially and, consequently, the behaviour is unstable (See Figure 2).

It can be shown by dynamic analysis that the travelling plate undergoes divergence instability at a sufficiently high speed [8] and thus it is sufficient 
to perform static analysis, i.e., study the case with $\tilde{s}=0$. (See also Figure 2.)

The stationary equations for $W$ are, substituting (9) into (3) and setting $\tilde{s}=0$,

$$
\left(m V_{0}^{2}-T\right) \frac{\partial^{2} W}{\partial x^{2}}+D\left(\frac{\partial^{4} W}{\partial x^{4}}+2 \frac{\partial^{4} W}{\partial x^{2} \partial y^{2}}+\frac{\partial^{4} W}{\partial y^{4}}\right)=0
$$

with boundary conditions $(6)-(8)$. We rewrite (10) as

$$
-\frac{\ell^{2}}{\pi^{2}}\left(\frac{\partial^{4} W}{\partial x^{4}}+2 \frac{\partial^{4} W}{\partial x^{2} \partial y^{2}}+\frac{\partial^{4} W}{\partial y^{4}}\right)=\lambda \frac{\partial^{2} W}{\partial x^{2}}
$$

where

$$
\lambda=\gamma^{2}=\frac{\ell^{2}}{\pi^{2} D}\left(m V_{0}^{2}-T\right)
$$

is the eigenvalue.

In order to determine the minimal eigenvalue (12) of the problem (6) (8), (11), and the corresponding eigenfunction $W=W(x, y)$, the following representation is applied:

$$
W=W(x, y)=f\left(\frac{y}{b}\right) \sin \left(\frac{\pi x}{\ell}\right)
$$

where $f(y / b)$ is an unknown function. Note that $W$ in (13) satisfies the boundary condition (6). The solution, a half-sine in the longitudinal direction, is well-known (see, e.g., [8]). Using the dimensionless formulation

$$
\eta=\frac{y}{b}, \quad \kappa=\frac{\ell}{\pi b}
$$

and the relations $(7)-(8)$ and $(10)-(14)$, the following eigenvalue problem 
for the unknown function $f(\eta)$ is obtained:

$$
\begin{aligned}
\kappa^{4} \frac{\mathrm{d}^{4} f}{\mathrm{~d} \eta^{4}}-2 \kappa^{2} \frac{\mathrm{d}^{2} f}{\mathrm{~d} \eta^{2}}+(1-\lambda) f=0, & -1<\eta<1 \\
\kappa^{2} \frac{\mathrm{d}^{2} f}{\mathrm{~d} \eta^{2}}-\nu f=0, & \eta= \pm 1 \\
\kappa^{2} \frac{\mathrm{d}^{3} f}{\mathrm{~d} \eta^{3}}-(2-\nu) \frac{\mathrm{d} f}{\mathrm{~d} \eta}=0, & \eta= \pm 1 .
\end{aligned}
$$

The problem $(15)-(17)$ is now considered as a spectral boundary value problem. The symmetric solution of $(15)$ - (17) corresponding to the minimal eigenvalue $\lambda$ is

$$
f(\eta)=A \cosh \left(\frac{\sqrt{1+\gamma}}{\kappa} \eta\right)+B \cosh \left(\frac{\sqrt{1-\gamma}}{\kappa} \eta\right)
$$

where $A$ and $B$ are arbitrary constants.

In [3] it was shown that the buckling mode is symmetric. When $\gamma \leq 1$, the symmetric divergence mode is thus

$$
W=f(\eta) \sin \left(\frac{\pi x}{\ell}\right)
$$

To determine the constants $A$ and $B$ in (18), we insert $f$ from equation (18) into the boundary conditions $(16)-(17)$ :

$$
\begin{gathered}
A(1+\gamma-\nu) \cosh \left(\frac{\sqrt{1+\gamma}}{\kappa}\right) \\
+B(1-\gamma-\nu) \cosh \left(\frac{\sqrt{1-\gamma}}{\kappa}\right)=0 \\
A \sqrt{1+\gamma}(1-\gamma-\nu) \sinh \left(\frac{\sqrt{1+\gamma}}{\kappa}\right) \\
+B \sqrt{1-\gamma}(1+\gamma-\nu) \sinh \left(\frac{\sqrt{1-\gamma}}{\kappa}\right)=0
\end{gathered}
$$


The condition for a non-trivial solution to exist in the form (18) - (19) is that the determinant of the system $(20)-(21)$ must vanish. This leads to the transcendental equation

$$
\Phi(\gamma, \kappa)-\Psi(\gamma, \nu)=0
$$

which determines the eigenvalues $\lambda=\gamma^{2}$ as an implicit function. In (22),

$$
\Phi(\gamma, \kappa)=\tanh \left(\frac{\sqrt{1-\gamma}}{\kappa}\right) \operatorname{coth}\left(\frac{\sqrt{1+\gamma}}{\kappa}\right)
$$

and

$$
\Psi(\gamma, \nu)=\frac{\sqrt{1+\gamma}}{\sqrt{1-\gamma}} \frac{(\gamma+\nu-1)^{2}}{(\gamma-\nu+1)^{2}} .
$$

Detailed analysis of the properties of $\Phi$ and $\Psi$ can be found in [10].

It follows that the critical velocity of instability of the travelling plate is given by

$$
V_{0}^{*}=\sqrt{\frac{T}{m}+\gamma_{*}^{2} \frac{\pi^{2} D}{m l^{2}}}
$$

where $\gamma_{*}$ is the root of the equation (22). The motion of the plate is stable when its velocity satisfies

$$
0 \leq V_{0}<V_{0}^{*}
$$

As the root $\gamma_{*}$ does not depend on the value of tension $T$, the limit of stable velocity (25) is increased when increasing the tension T. However, in the case of a cracked plate, increasing tension may lead to the growth of the crack.

2.2. Probabilistic analysis of critical velocity considering instability and fracture

In the following, we include uncertainty in the parameters of the plate and formulate the problem of finding the critical velocity of the plate un- 
der constraints for the probabilities of instability and fracture. Introducing a constraint for the probability of failure is a way to formulate statistical mechanical problems, used by many researchers (see, e.g., [26, 27]).

Let $(\Omega, \mathcal{F}, \mathbb{P})$ be a probability space, where $\Omega$ is a sample space, $\mathcal{F}$ is its $\sigma$-algebra and $\mathbb{P}$ is a probability measure on $\mathcal{F}$. Let $\xi, h, m, \nu, E$, the critical strain energy release rate, $G_{\mathrm{C}}$, and the solution of the equation $(22), \gamma_{*}$, be random variables on $\Omega$, the image of each random variable being an interval of $\{x \in \mathbb{R}: x>0\}$. The tension applied to the supported edges of the plate is defined as

$$
T=T_{0}(1+\theta)
$$

where $T_{0}$ is a positive constant and $\theta$ is a random variable on $\Omega$ with

$$
-1<\theta(\omega)<1, \quad \omega \in \Omega
$$

The stress intensity factors $K_{j}, j=I, I I$, related to the crack modes $I$ (opening) and $I I$ (in-plane shear) have the form

$$
K_{j}=\frac{\alpha_{j}(\xi) T \sqrt{\pi \xi}}{h}, \quad j=I, I I,
$$

and are random variables. In $(29), \alpha_{j}$ is a weight function, the expression of which depends on the crack geometry. Formulae for weight functions $\alpha_{j}$ are presented, e.g., in [28], [29], [30] and [31]. In this study, the weight functions $\alpha_{j}$ are assumed to be strictly positive, continuous and increasing.

The fracture toughness $K_{\mathrm{C}}$ of the material is given by

$$
K_{\mathrm{C}}=\sqrt{G_{\mathrm{C}} E}
$$

and is also a random variable. 
Considering both instability and fracture, the problem of critical velocity of the plate in the presence of a mode $I$ crack reads as

$$
\begin{aligned}
& \max _{T} V_{0} \text { such that } \\
& \mathbb{P}\left(K_{I} \geq K_{\mathrm{C}}\right) \leq p_{\mathrm{f}} \text { and } \\
& \mathbb{P}\left(V_{0} \geq \sqrt{\frac{T}{m}+\gamma_{*} \frac{\pi^{2} D}{m \ell^{2}}}\right) \leq p_{\mathrm{i}},
\end{aligned}
$$

where $p_{\mathrm{i}}, p_{\mathrm{f}}$ denote the upper bounds of the probabilities of instability and fracture, respectively. In the case of a mixed mode crack, the constraint (32) is replaced by

$$
P\left(K_{I}^{2}+1.56 K_{I I}^{2} \geq K_{\mathrm{C}}^{2}\right) \leq p_{\mathrm{f}} .
$$

For the failure criterion (34) of a mixed mode crack, see [32, 33].

Using (29), the constraint (34) is equivalent to

$$
\mathbb{P}\left(\frac{\alpha(\xi) T \sqrt{\pi \xi}}{h} \geq K_{\mathrm{C}}\right) \leq p_{\mathrm{f}}
$$

with

$$
\alpha(\xi)=\sqrt{\left(\alpha_{I}(\xi)\right)^{2}+1.56\left(\alpha_{I I}(\xi)\right)^{2}} .
$$

In the case of a mode I crack we denote

$$
\alpha=\alpha_{I} .
$$

The inequalities (32) and (34) are equivalent to

$$
F_{X}\left(T_{0}\right) \leq p_{\mathrm{f}}
$$

where $F_{X}$ is the cumulative distribution function of the random variable $X$,

$$
X=\frac{K_{\mathrm{C}} h}{\alpha(\xi) \sqrt{\pi \xi}(1+\theta)} .
$$


The function $F_{X}$ is continuous and strictly increasing, when

$$
T_{0} \in\left[\inf _{\Omega} X, \sup _{\Omega} X\right],
$$

and

$$
F_{X}\left(\inf _{\Omega} X\right)=0, \quad F_{X}\left(\sup _{\Omega} X\right)=1 .
$$

Thus the maximal value of $T_{0}$ that satisfies (32) (or (34)), denoted by $T_{0}^{\mathrm{cr}}$, is found on the interval in (40) by solving the equation

$$
F_{X}\left(T_{0}\right)-p_{\mathrm{f}}=0
$$

and is the $p_{\mathrm{f}}$ th order quantile of $F_{X}$ :

$$
T_{0}^{\mathrm{cr}}=F_{X}^{-1}\left(p_{\mathrm{f}}\right) .
$$

The inequality (33) is equivalent to

$$
F_{Y_{T_{0}}}\left(V_{0}\right) \leq p_{\mathrm{i}}
$$

where

$$
Y_{T_{0}}=\sqrt{\frac{T_{0}(1+\theta)}{m}+\gamma_{*} \frac{\pi^{2} D}{m \ell^{2}}} .
$$

The cumulative distribution function $F_{Y_{T_{0}}}$ is continuous, increasing with respect to $V_{0}$ and decreasing with respect to $T_{0}$. Hence the maximum value of $V_{0}$, such that (33) and (32) (or (34)) are satisfied, is found by studying

$$
F_{Y}, \quad Y:=Y_{T_{0}^{\text {cr }}} .
$$

The solution of the problem (31)-(33) (or the problem (31), (33) and (34)), denoted by $V_{0}^{\text {cr }}$, is the $p_{\mathrm{i}}$ th order quantile of $F_{Y}$ :

$$
V_{0}^{\mathrm{cr}}=F_{Y}^{-1}\left(p_{\mathrm{i}}\right) .
$$


Table 1 shows the solutions of the problems in which only the crack length, the tension variation and the strain energy release rate or one of them is regarded as a random variable. When the only random valued parameter is $\xi$ or $G_{\mathrm{C}}$, there are no random variables in the constraint (33). In this case, the critical velocity is simply given by (25). In Table 1 ,

$$
F_{j}, \quad j=\xi, \theta, G_{\mathrm{C}}
$$

is the cumulative distribution function of the random variable $j$.

\subsection{Numerical approximation of the quantiles}

If the distributions of the random valued problem parameters do not enable an analytical expression for the quantile function $F^{-1}$ appearing in the formula of the critical tension or velocity, its approximative values can be obtained by Monte Carlo simulation. When approximating $F^{-1}(p)$ with a given $p$, the sampling from $F$ needs to be done only once. Indeed, let $S_{1}, \ldots, S_{n}$ be a random sample from the distribution $F$, where $n$ is the sample size. The order statistics of the sample are

$$
S_{1}^{(n)} \leq S_{2}^{(n)} \leq \cdots \leq S_{n}^{(n)}
$$

and the $p$ th quantile $F^{-1}(p)$ can be approximated as

$$
F^{-1}(p) \approx S_{\lceil n p\rceil}^{(n)},
$$

where $\lceil n p\rceil$ is the first integer $\geq n p$. In [34] it is shown that $S_{\lceil n p\rceil}^{(n)}$ is a weakly consistent quantile estimator.

With the sample $S_{1}, \ldots, S_{n}$ from the distribution $F$, the strongly consistent estimator of $F\left(T_{0}\right)$ is

$$
\hat{F}_{n}\left(T_{0}\right)=\frac{\sum_{k=1}^{n} \chi_{\left\{S_{k} \leq T_{0}\right\}}}{n},
$$


where $\chi_{A}$ is the indicator of the event $A$, and its standard error is

$$
\text { s.e. }\left(\hat{F}_{n}\left(T_{0}\right)\right)=\sqrt{\frac{\hat{F}_{n}\left(T_{0}\right)\left(1-\hat{F}_{n}\left(T_{0}\right)\right)}{n}} .
$$

The estimator (50) and its standard error (51) are used for evaluating the sufficient sample size in quantile estimation. By setting

$$
\frac{s . e .\left(\hat{F}_{n}\left(T_{0}\right)\right)}{\hat{F}_{n}\left(T_{0}\right)} \leq r
$$

where $r \in(0,1)$, a lower bound for the sample size $n$ in estimating the $p$ th order quantile $F^{-1}(p)$ is obtained:

$$
n \geq \frac{1-p}{p r^{2}}
$$

The values of the quantiles $F_{\theta}^{-1}$ and $F_{G_{\mathrm{C}}}^{-1}$, with normally distributed $\theta$ and $G_{\mathrm{C}}$, can be obtained by a subroutine of a standard program library (e.g. SciPy). For a Weibull distributed $\xi$, an analytical expression for $F_{\xi}^{-1}$ exists.

\section{Numerical results and discussion}

In this section, the solutions obtained in Sections 2.2 and 2.3 are illustrated in a paper industrial context.

The random variables $\xi, h, \nu, E, G_{\mathrm{C}}$ and $\theta$ were assumed to be independent. The random variables $h, \nu, E, G_{\mathrm{C}}, \theta$, were assumed to obey the normal distribution with the means $\mu_{j}, j=h, \nu, E, G_{\mathrm{C}}, \theta$, in Table 2. The mean values were chosen to correspond to the properties of printing paper. The mass of the plate $m$ was assumed to be dependent of $h$ :

$$
m(\omega)=\frac{\mu_{m}}{\mu_{h}} h(\omega), \quad \omega \in \Omega
$$


The effect of variation in the parameter values on the solution was studied by introducing the coefficient of variation, $\mathrm{CV}_{j}$, for the random variable $j$ as a relation of its standard deviation, $\sigma_{j}$, and mean, $\mu_{j}$ :

$$
\mathrm{CV}_{j}=\frac{\sigma_{j}}{\mu_{j}}
$$

The crack length was assumed to be distributed according to the Weibull distribution with a shape parameter $s \in(0,1]$. The cumulative distribution function of the Weibull distributed crack length is

$$
F_{\xi}(x)=\int_{0}^{x} \frac{s}{c}\left(\frac{t}{c}\right)^{s-1} e^{-(t / c)^{s}} d t=1-e^{-(x / c)^{s}},
$$

where $x \geq 0$ and the scale parameter $c>0$. The expected value and variance of the crack length are

$$
\mathrm{E}[\xi]=c \Gamma\left(1+\frac{1}{s}\right), \quad \operatorname{Var}[\xi]=c^{2}\left(\Gamma\left(1+\frac{2}{s}\right)-\left(\Gamma\left(1+\frac{1}{s}\right)\right)^{2}\right),
$$

where $\Gamma$ is the gamma function,

$$
\Gamma(x)=\int_{0}^{\infty} t^{x-1} e^{-t} d t .
$$

When the shape parameter $s \in(0,1]$, the probability density function is strictly decreasing. For $s \in(0,1)$, the probability density function tends to infinity when $x$ tends to zero. For $s=1$, the probability density function tends to $1 / c$ when $x$ tends to zero, the distribution corresponding to the exponential distribution with intensity $1 / c$. Figure 3 shows the effect of changing the value of $c$ on the probability density function. It shows that when $c$ is decreased, the probability mass becomes more concentrated near 
the origin, corresponding to the small values of the crack length. When $c$ is decreased, both the expected value and variance of the crack length decrease. The shape parameter $s$ has the opposite effect on the probability density function, as is seen in Figure 3.

In cases where the problem parameters $m, h, \nu, E$ and $G_{\mathrm{C}}$ were regarded as constants, their values were set to the mean values in Table 2 . If the crack length was regarded as a constant, its value was set to be equal to the expected crack length of the stochastic model to which it is compared. In all computations, the geometrical constants of the plate were given constant values $\ell=0.1(\mathrm{~m})$ and $b=5(\mathrm{~m})$.

The problem was studied with an edge crack parallel to the $y$ direction. The weight function $\alpha$ was approximated with the constant function

$$
\alpha \equiv 1.12
$$

In [22] critical velocities with different crack geometries were compared. When the weight functions are approximated with constant functions, the perpendicular edge crack model gives lower critical velocity than the central crack model, if the distributions of the cracks are the same. This is, naturally, due to the higher value of the weight function of the edge crack.

In approximating the quantiles (43) and (47), the sample size $n=2 \cdot 10^{6}$ was used in Monte Carlo simulation for all $p_{\mathrm{i}}, p_{\mathrm{f}}$ of interest. This sample size leads the relative error of the cumulative distribution function estimator, expressed in (52), to be less than $2.5 \%$ for $p_{\mathrm{f}}, p_{\mathrm{i}}=0.001$.

The model in which $m, h, \nu, E, G_{\mathrm{C}}, \xi$ and $\theta$ are regarded as random variables was compared with the model in which only the parameters $G_{\mathrm{C}}, \xi$ 
and $\theta$ are random. The difference of the models was studied with

$$
\left.\left.\mathrm{CV}_{h}, \mathrm{CV}_{E} \in\right] 0,0.05\right], \quad \mathrm{CV}_{\nu} \in\{0.01,0.05\}
$$

The parameters of the common random variables of the models were set as $\mathrm{CV}_{\theta}=0.1, \mathrm{CV}_{G_{\mathrm{C}}}=0.01, c=0.005$ and $s=0.5$, and the probabilities of fracture and instability were $p_{\mathrm{f}}=p_{\mathrm{i}}=0.001,0.1$. The critical velocities obtained with the first mentioned model were somewhat lower than the ones obtained by the other model but the difference may not be regarded as significant (2\% lower at maximum). Thus the results suggest that at least small variation in the thickness and mass of the plate or in the elastic coefficients is not a significant factor, when the critical velocity of the cracked thin plate is studied.

In Figure 5 the critical velocities given by the model with random $G_{\mathrm{C}}, \theta$ and $\xi$ and the models with a single random parameter are compared to the critical velocity given by the corresponding fully deterministic model. The critical velocities were thus divided by the coefficient $\tilde{V}_{0}$,

$$
\tilde{V}_{0}=\sqrt{\frac{1}{\mu_{m}}\left(\tilde{T}_{0}+\tilde{\gamma}_{*} \frac{\pi^{2} \tilde{D}}{\ell^{2}}\right)},
$$

where

$$
\tilde{T}_{0}=\frac{\mu_{h} \tilde{K}_{\mathrm{C}}}{\alpha(\mathrm{E}[\xi]) \sqrt{\pi \mathrm{E}[\xi]}}
$$

$\tilde{\gamma}_{*}$ is the solution of the equation (22) with $\nu=\mu_{\nu}$ and $\kappa=\ell / \pi \mu_{b}$,

$$
\tilde{D}=\frac{\mu_{E} \mu_{h}^{3}}{12\left(1-\mu_{\nu}^{2}\right)} \quad \text { and } \quad \tilde{K}_{\mathrm{C}}=\sqrt{\mu_{G_{\mathrm{C}}} \mu_{E}} .
$$

The crack length distribution parameters were set as $s=0.5$ and $c=0.005$. The coefficient $\tilde{V}_{0}$ is the critical velocity of the fully deterministic model with 
a crack of the length $\mathrm{E}[\xi]=0.01(\mathrm{~m})$. The relative critical velocity of the fully deterministic model becomes equal to unity.

Figure 5 shows that, with a fixed reliability level, among the models with a single random valued parameter, the model with random crack length produces the lowest critical velocity. The model with random strain energy release rate provides critical values that are the closest to the critical values by the deterministic model. It is seen that, with low $C V_{G_{\mathrm{C}}}$, variation in strain energy release rate may not be significant, the size of the effect depending on the probability $p_{\mathrm{f}}$. The effect of variation in tension is more significant than the impact of variation in strain energy release rate.

The lowest critical velocity in Figure 5 is given by the model with random strain energy release rate, tension and crack length. The effect of $C V_{\theta}$ and $C V_{G_{\mathrm{C}}}$ on the relative critical velocity was studied in the range $C V_{\theta}, C V_{G_{\mathrm{C}}} \in$ $[0,0.2]$, with fixed crack length distribution $(s=0.5, c=0.005)$. A change in $C V_{G_{\mathrm{C}}}$ had no effect on the relative critical velocity but increasing $C V_{\theta}$ decreases the critical velocity, although this effect may not be significant with low $C V_{\theta}$.

The above results suggest that, when the effect of variation in the problem parameters is considered, the most significant factor is the random crack length. Including also variation of tension in the model may lower the critical velocity notably, at least if the variation is remarkable. The results agree with the study by Uesaka [1] to some extent. According to [1], the majority of web breaks in paper production are caused by tension variations, combined with strength variations of the paper web. The results of the present study suggest that variation in tension is significant, but variation in strain energy 
release rate, which causes variation in paper fracture toughness, is not.

Figure 3 shows that a change in the scale parameter $c$ does not affect the relative critical velocity, when the critical velocity obtained with the model of random crack length is compared to the critical velocity of the corresponding deterministic model. The effect of $c$ was studied on the interval ]0,0.01], and it is seen in the figure that the relative critical velocity remains almost constant when $c$ is changed. Figure 3, in turn, shows that a change in the shape parameter $s$ has an effect on the relative critical velocity. The figures also show the expected value and standard deviation of the crack length $\xi$ with respect to the distribution parameters. In Figure 3 it is seen that as the expected value and standard deviation of the crack length increase, the relative critical velocity decreases.

Figure 8 shows the relative critical velocity by the random crack length model with respect to $p_{\mathrm{f}}$ for some values of $s$. When $s$ decreases, the value of $p_{\mathrm{f}}$ at which the critical velocity given by the stochastic model coincides with the critical velocity by the deterministic model also decreases. However, when $s$ decreases, the mean and standard deviation of the crack length increase. With small mean crack length and by demanding the probability of fracture to be low, the stochastic model produces considerably lower critical velocity than the deterministic model.

Some of the critical values of tension and velocity given by the model with random crack length and the model with both random crack length and tension are gathered in Table 3. The obtained critical velocities are higher than the running speeds of the current paper machines. However, it should be noted that the surrounding air is excluded from the model. The presence of 
surrounding air is known to influence the critical velocity [35, 36]. According to [35], the critical velocity obtained with the vacuum model may be even four times the critical value by the model that includes the surrounding air.

The parameter values were chosen to correspond to those of a dry paper web. If wet paper is modelled, also material properties such as viscoelasticity should be included in the model. However, the effect of viscoelasticity on the critical stable velocity is found small (see [11, 12]). Another excluded material property, independent of the moisture of paper, is orthotropicity. As with viscoelasticity, the effect of orthotropicity on the critical stable velocity is found small (see $[3,10,37])$. From the viewpoint of the application, another notable simplification considers the tension profile. In this study, the profile of tension was assumed to be homogeneous, although in paper machines, the measured tension varies in the cross direction [38].

\section{Conclusions}

In this study, stochastic analysis of the critical velocity of an axially moving elastic and isotropic plate in the presence of a crack was presented. The critical velocity was derived under constraints for the probabilities of instability and fracture, which are the most critical threats to runnability of axially moving materials.

The solution was illustrated with a paper industrial example. The crack length was assumed to obey the Weibull distribution while the other random valued problem parameters were assumed to obey the normal distribution.

Altering the set of random parameters in the model, the effect of variation in problem parameters on the critical velocity was studied. The critical 
velocity obtained by the model where the random parameters included the mass and thickness of the plate, the elastic coefficients, the strain energy release rate, the tension to which the plate is subjected, and the crack length, was compared with the model with only random strain energy release rate, tension and crack length. The results showed no significant effect of introducing variation in the values of the mass and thickness of the plate or in the elastic coefficients.

When the models in which only the strain energy release rate, tension or crack length is regarded as a random variable were compared, the lowest critical velocity was produced by the random crack length model. The effect of variation in strain energy release rate was not found significant, at least, if the dispersion is not remarkable. Compared with a fully deterministic model, the model with random crack length produced significantly lower critical values, the size of the effect depending on the values of the constraining probabilities and distribution parameters. Introducing variation of tension in the random crack length model decreased the critical velocity, and the impact of tension variation was significant with high dispersion.

The results suggest that when the critical velocity of the cracked plate is studied, it is essential to include variation in crack length, and possibly also variation in tension magnitude, in the model. Randomness of other problem parameters is not significant, and the model can be simplified such that the random parameters include only the crack length and tension. 


\section{Acknowledgements}

This research was supported by the Academy of Finland (grant no. 140221), the KAUTE Foundation and the Jenny and Antti Wihuri Foundation.

\section{References}

\section{References}

[1] Uesaka T. Web Breaks in Pressroom: A Review. FSCN-rapport; FSCN, Mitthögskolan; $2004 . \quad$ URL http://books.google.fi/books?id=xs6YMQAACAAJ.

[2] Wathén R. Characterizing the influence of paper structure on web breaks. Licentiate thesis; Helsinki University of Technology, Department of Forest Products Technology; 2003. Espoo, Finland.

[3] Banichuk N, Jeronen J, Neittaanmäki P, Tuovinen T. On the instability of an axially moving elastic plate. International Journal of Solids and Structures 2010;47(1):91-9. URL http://dx.doi.org/10.1016/j.ijsolstr.2009.09.020.

[4] Skutch R. Uber die Bewegung eines gespannten Fadens, weicher gezwungen ist durch zwei feste Punkte, mit einer constanten Geschwindigkeit zu gehen, und zwischen denselben in Transversal-schwingungen von gerlinger Amplitude versetzt wird. Annalen der Physik und Chemie 1897;61:190-5.

[5] Sack RA. Transverse oscillations in traveling strings. British Journal of Applied Physics 1954;5:224-6. 
[6] Archibald FR, Emslie AG. The vibration of a string having a uniform motion along its length. ASME Journal of Applied Mechanics $1958 ; 25: 347-8$.

[7] Wickert JA, Mote CD. Classical vibration analysis of axially moving continua. ASME Journal of Applied Mechanics 1990;57:738-44.

[8] Lin CC. Stability and vibration characteristics of axially moving plates. International Journal of Solids and Structures 1997;34(24):3179-90.

[9] Marynowski K. Non-linear vibrations of the axially moving paper web. Journal of Theoretical and Applied Mechanics 2008;46(3):565 - 80 .

[10] Banichuk N, Jeronen J, Kurki M, Neittaanmäki P, Saksa T, Tuovinen T. On the limit velocity and buckling phenomena of axially moving orthotropic membranes and plates. International Journal of Solids and Structures 2011;48(13):2015-25. URL http://dx.doi.org/10.1016/j.ijsolstr.2011.03.010.

[11] Marynowski K. Free vibration analysis of the axially moving Levytype viscoelastic plate. European Journal of Mechanics - A/Solids 2010;29(5):879 -86. DOI: 10.1016/j.euromechsol.2010.03.010.

[12] Saksa T, Banichuk N, Jeronen J, Kurki M, Tuovinen T. Dynamic analysis for axially moving viscoelastic panels. International Journal of Solids and Structures 2012;49(23-24):3355-66. URL http://dx.doi.org/10.1016/j.ijsolstr.2012.07.017.

[13] Sobczyk K. Free vibrations of elastic plate with random properties The eigenvalue problem. Journal of Sound and Vibration 1972;22:33-9. 
[14] Wood A, Zaman F. Free vibrations of randomly inhomogeneous plates. Journal of Sound and Vibration 1977;52:543-52.

[15] Soares CG. Uncertainty modelling in plate buckling. Structural Safety 1988;5:17-34.

[16] Irwin GR. Fracture. In: Flugge S, editor. Handbuch der Physik; vol. VI. Berlin: Springer-Verlag; 1958, p. 551-90.

[17] Inglis CE. Stresses in a plate due to the presence of cracks and sharp corners. Trans Institute of Naval Architecture 1913;55:219-41.

[18] Griffith AA. The phenomena of rupture and flow in solids. Philosophical Transactions of the Royal Society of London 1921;A 221:163-98.

[19] Westergaard HM. Bearing pressures and cracks. Journal of Applied Mechanics 1939;6:A49-53.

[20] Banichuk N, Kurki M, Neittaanmäki P, Saksa T, Tirronen M, Tuovinen T. Optimization and analysis of processes with moving materials subjected to fatigue fracture and instability. Mechanics Based Design of Structures and Machines: An International Journal 2013;41(2):146-67. URL http://dx.doi .org/10.1080/15397734.2012.708630.

[21] Banichuk N, Jeronen J, Neittaanmäki P, Saksa T, Tuovinen T. Mechanics of moving materials; vol. 207 of Solid mechanics and its applications. Springer; 2014. ISBN: 978-3-319-01744-0 (print), 978-3-31901745-7 (electronic). 
[22] Tirronen M, Tuovinen T, Jeronen J, Saksa T. Stochastic analysis of the critical stable velocity of a moving paper web in the presence of a crack. In: I' Anson SJ, editor. Advances in Pulp and Paper Research, Cambridge 2013; vol. 1. The Pulp \& Paper Fundamental Research Society; 2013, p. 301-19. ISBN: 978-0-9926163-0-4.

[23] Bolotin VV. Nonconservative Problems of the Theory of Elastic Stability. New York: Pergamon Press; 1963.

[24] Timoshenko SP, Woinowsky-Krieger S. Theory of plates and shells. New York : Tokyo : McGraw-Hill; 2nd ed.; 1959. ISBN 0-07-085820-9.

[25] Leissa AW. The free vibration of rectangular plates. Journal of Sound and Vibration 1973;31(3):257-93. URL http://dx .doi .org/10.1016/S0022-460X (73)80371-2.

[26] Banichuk NV, Neittaanmäki PJ. Structural Optimization with Uncertainties. Dordrecht: Springer-Verlag; 2010. ISBN 978-90-481-2517-3.

[27] Bolotin V. Statistical Methods in Structural Mechanics. Holden-Day; 1969.

[28] Perez N. Fracture Mechanics. Kluwer Academic Publishers; 2004.

[29] Laham SA. Stress Intensity Factor and Limit Load Handbook. British Energy Generation Ltd; 1998.

[30] Fett T. Stress Intensity Factors, T-Stresses, Weight Functions. Supplement Volume. KIT Scientific Publishing; 2009. ISBN 978-3-86644-446-1. 
[31] Fett T. Stress Intensity Factors, T-Stresses, Weight Functions. Universitätsverlag Karlsruhe; 2008. ISBN 978-3-86644-235-1.

[32] Kundu T. Fundamentals of fracture mechanics. CRC Press; 2008. ISBN 978-0849384325.

[33] Zehnder A. Fracture Mechanics; vol. 60 of Lecture Notes in Applied and Computational Mechanics. Springer; 2012. ISBN 978-94-007-2594-2 (print) 978-94-007-2595-9 (online).

[34] Resnick SI. A Probability Path. Birkhäuser; 2005.

[35] Pramila A. Sheet flutter and the interaction between sheet and air. TAPPI Journal 1986;69(7):70-4.

[36] Frondelius T, Koivurova H, Pramila A. Interaction of an axially moving band and surrounding fluid by boundary layer theory. Journal of Fluids and Structures 2006;22(8):1047-56.

[37] Tuovinen T. Analysis of stability of axially moving orthotropic membranes and plates with linear non-homogeneous tension profile. Ph.D. thesis; Department of Mathematical Information Technology, University of Jyväskylä; 2011. Jyväskylä studies in computing 147. ISBN 978-951-39-4577-0 (book), ISBN 978-951-39-4578-7 (PDF); URL http://julkaisut.jyu.fi/?id=978-951-39-4578-7.

[38] Linna H, Parola M, Virtanen J. Better productivity by measuring web tension profile. In: 55th APPITA Annual Conference, Hobart, Australia 30 April-2 May 2001: Proceedings. Carlton, Vic.: Appita Inc.; 2001, p. 305-11. ISBN 0958554838. 


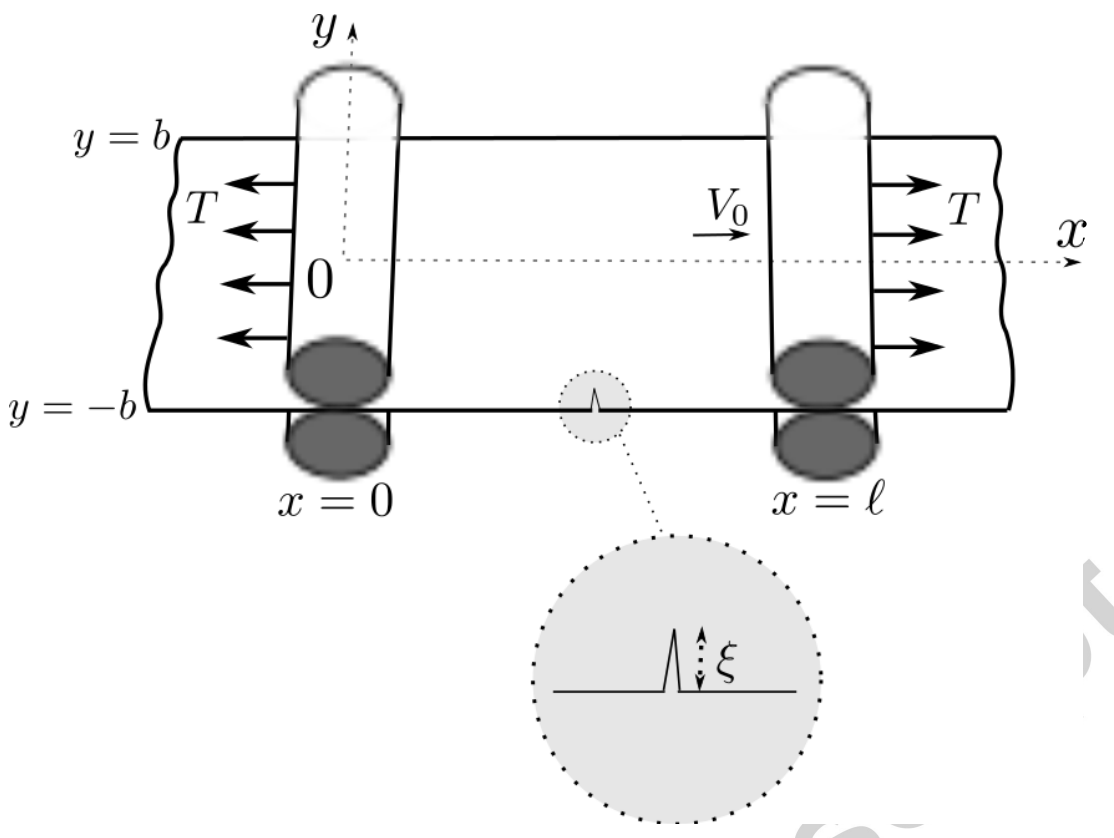

Figure 1: A travelling elastic plate with a crack.

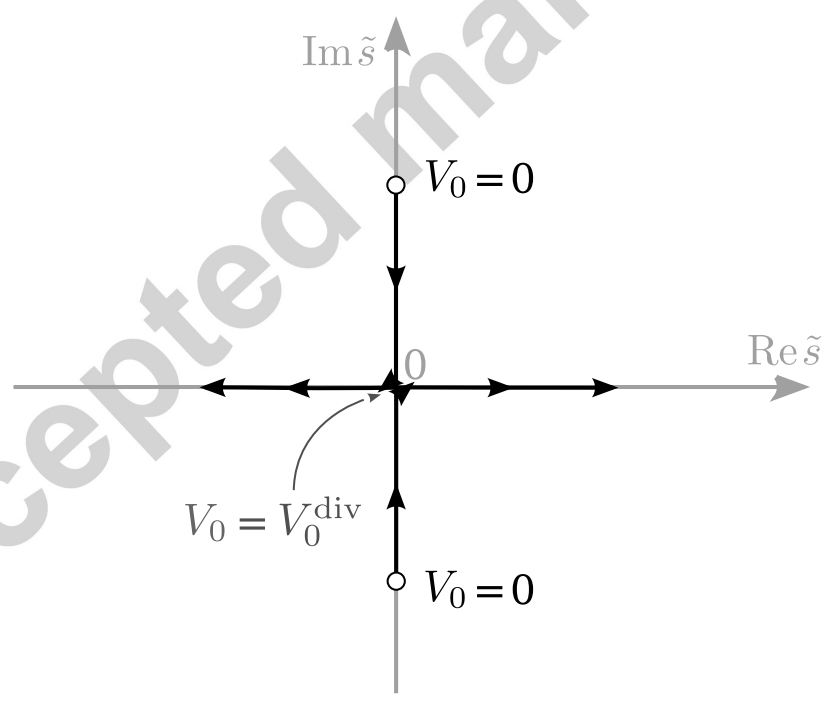

Figure 2: Behavior of the stability index $\tilde{s}$. Divergence, corresponding to the critical velocity, can be found at the origin. 


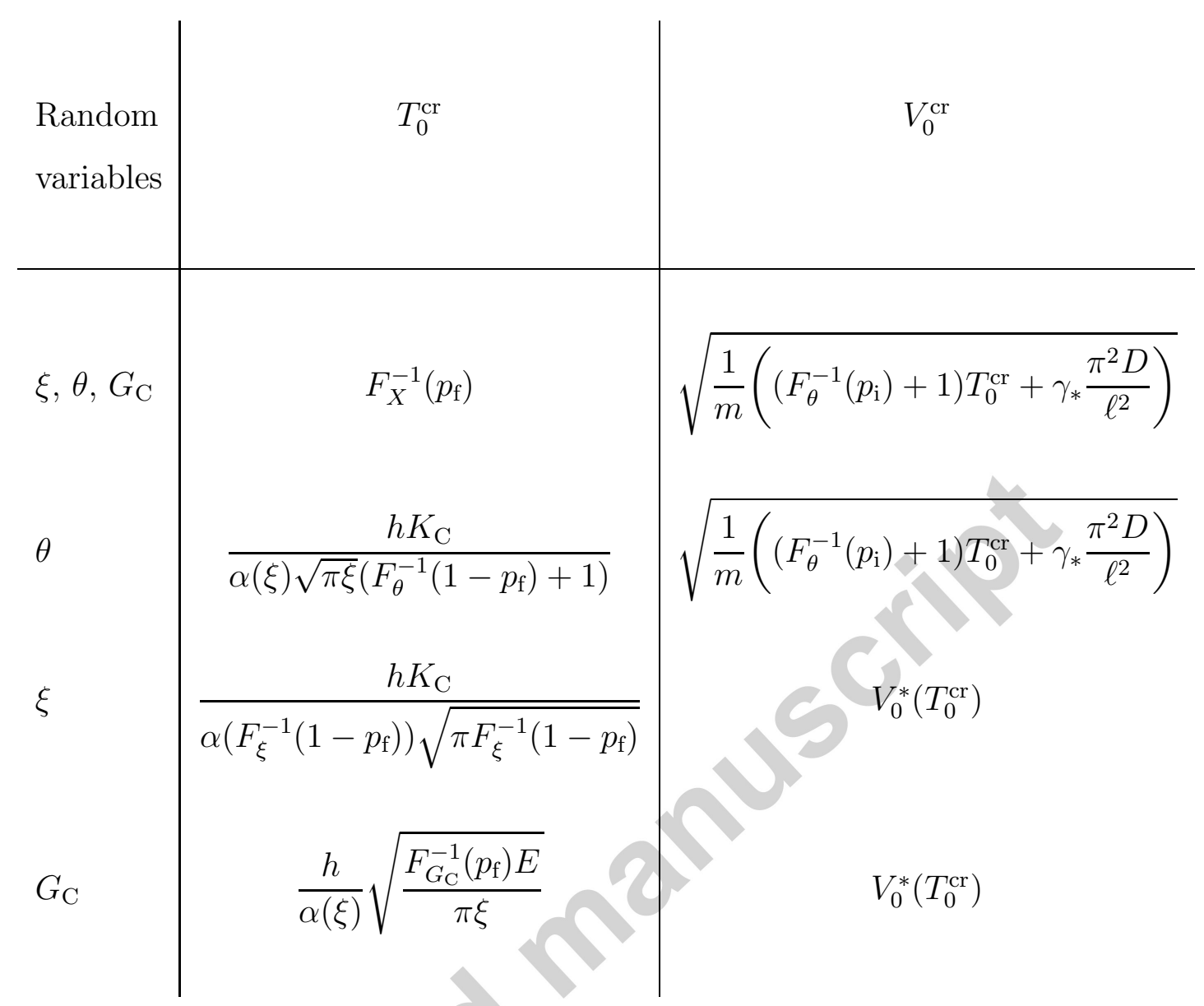

Table 1: Critical tensions and velocities for the problems in which only $\xi, \theta$ and $G_{\mathrm{C}}$ or one of them is a random variable.

\begin{tabular}{|l|l|}
\hline Parameter & $\mu$ \\
\hline$m$ & $0.08\left(\mathrm{~kg} / \mathrm{m}^{2}\right)$ \\
$h$ & $10^{-4}(\mathrm{~m})$ \\
$\nu$ & 0.3 \\
$E$ & $10^{9}(\mathrm{~Pa})$ \\
$G_{\mathrm{C}}$ & $8000\left(\mathrm{~J} / \mathrm{m}^{2}\right)$ \\
\hline
\end{tabular}

Table 2: Mean values of the parameters. 


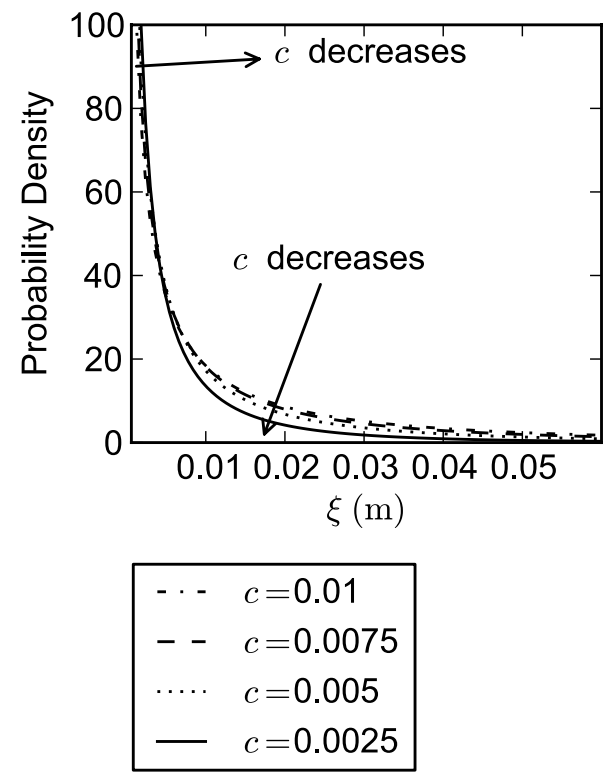

Figure 3: Weibull distribution. The effect of changing the value of the distribution parameter $c$ on the probability density function, $s=0.5$. 


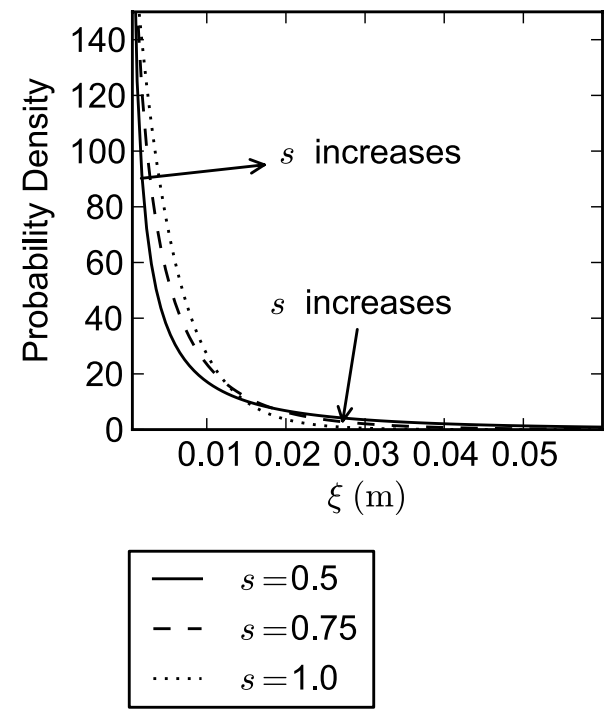

Figure 4: Weibull distribution. The effect of changing the value of the distribution parameter $s$ on the probability density function, $c=0.005$. 


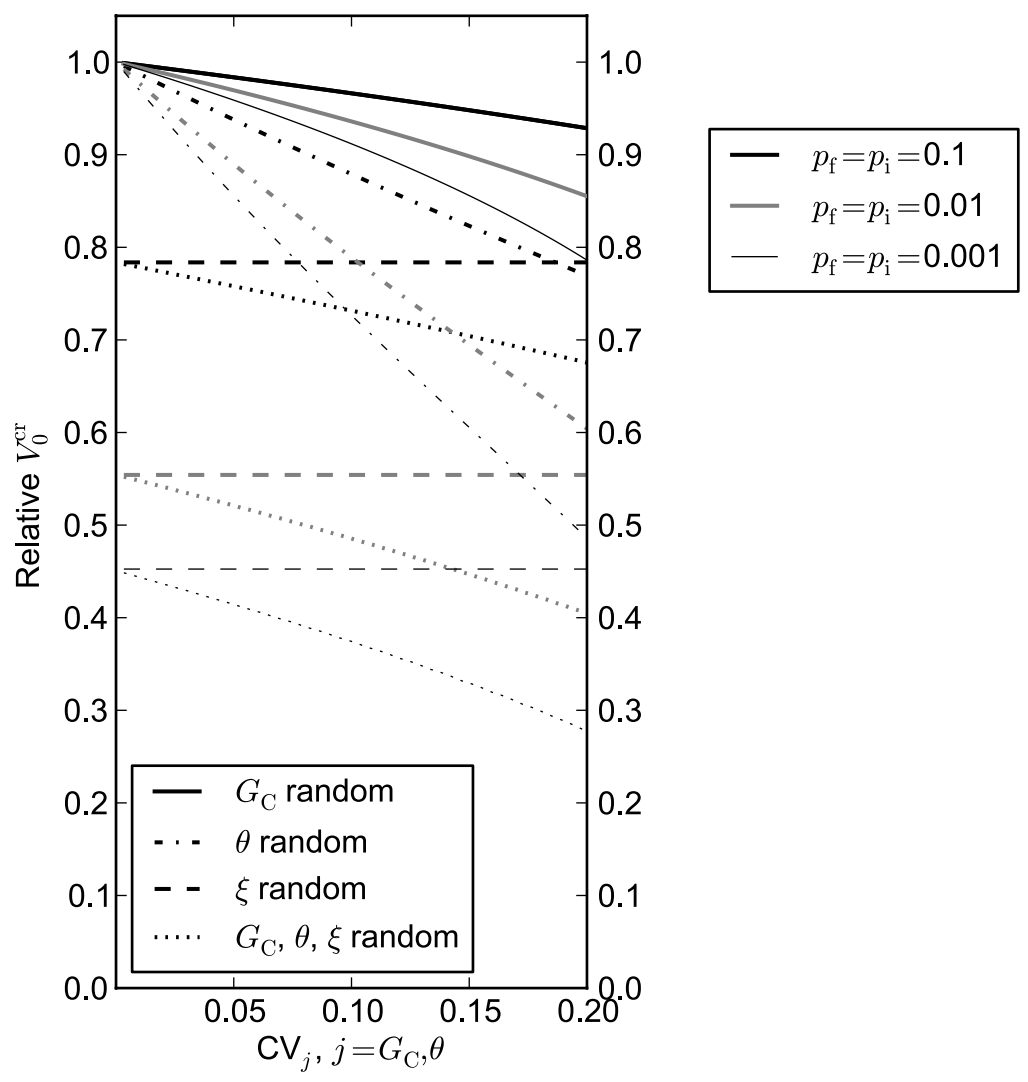

Figure 5: The relative critical velocities of the models in which crack length, tension variation and strain energy release rate or one of them is random, $s=0.5, c=0.005$. 


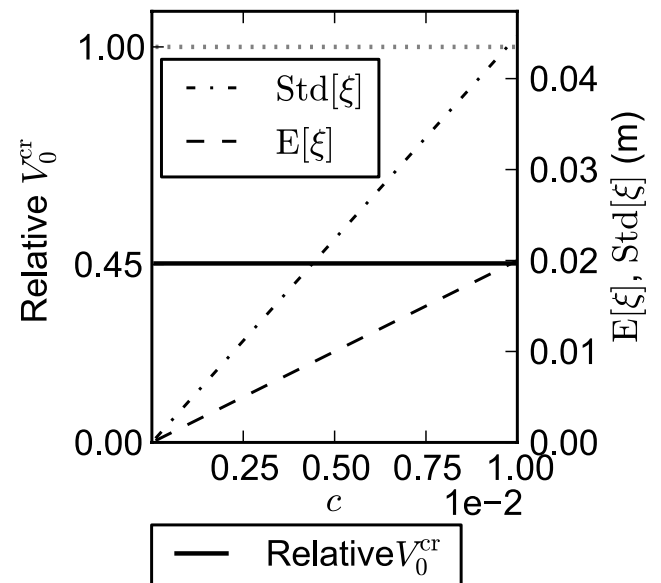

Figure 6: Random crack length. The relative critical velocity (the critical velocity of the model with random crack length divided by the critical velocity of the deterministic model) and the expected value and standard deviation of the crack length with respect to the distribution parameter $c, s=0.5, p_{\mathrm{f}}=0.001$. 


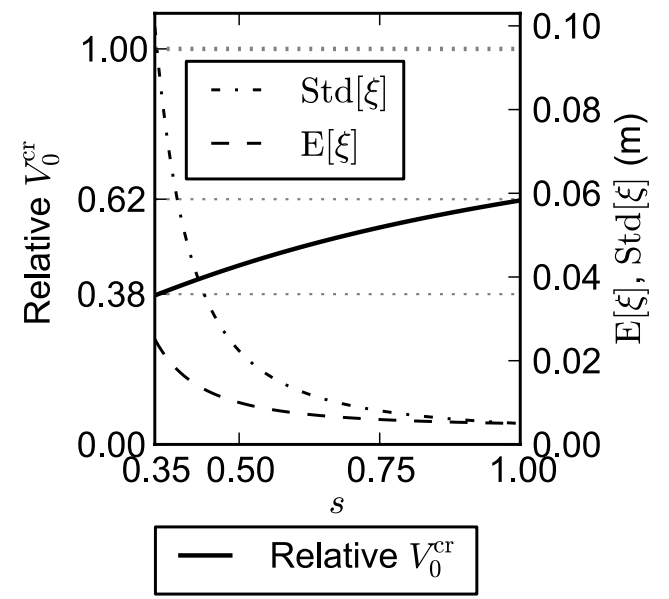

Figure 7: Random crack length. The relative critical velocity (the critical velocity of the model with random crack length divided by the critical velocity of the deterministic model) and the expected value and standard deviation of the crack length with respect to the distribution parameter $s, c=0.005, p_{\mathrm{f}}=0.001$. 




Figure 8: Random crack length. The relative critical velocity (the critical velocity of the model with random crack length divided by the critical velocity of the deterministic model) with respect to the probabilities $p_{\mathrm{f}}$ and $p_{\mathrm{i}}, c=0.005$. 


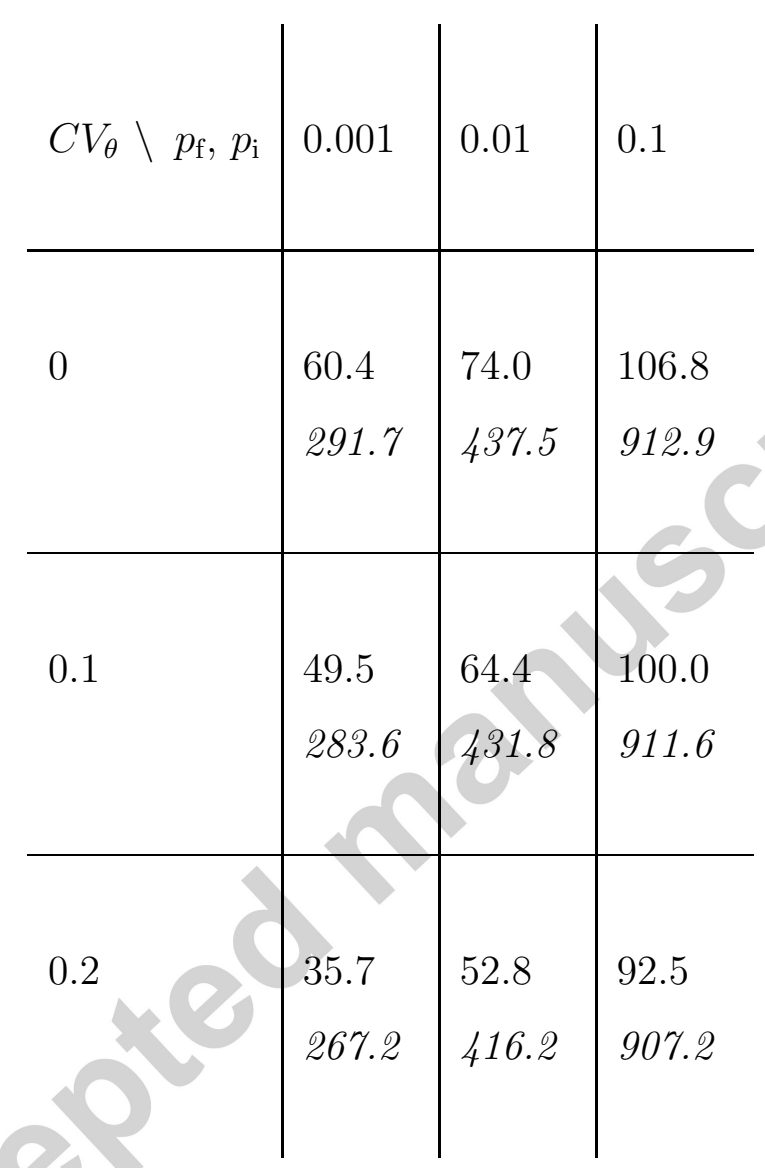

Table 3: The critical velocity (upper value, $\mathrm{m} / \mathrm{s}$ ) and the corresponding critical tension (lower value, $\mathrm{N} / \mathrm{m}$ ) with $s=0.5$ and $c=0.005$ with respect to $p_{\mathrm{f}}, p_{\mathrm{i}}$. 